Ann. Sci. forest., 1978, 35 (2), 139-150.

\title{
Relations entre groupes écologiques et propriétés physico-chimiques des sols brunifiés des Vosges cristallines
}

\author{
Martine GUILLEMIN *, J. ROUILLER et S. BRUCKERT \\ Centre de Pédologie Biologique, \\ 54500 Vandœurre-les-Nancy
}

\begin{abstract}
Résumé
Les auteurs présentent une série de déterminations physiques (taux d'agrégats stables, perméabilité) et physico-chimiques (CEC à divers $\mathrm{pH}$ ) qui établissent des relations hautement significatives entre la végétation (groupes écologiques) et les sols brunifiés de type ocreux, acide ou colluvial rencontrés sur une même pente de roches cristallines à l'étage montagnard.

Pour les deux extrêmes, sol brun ocreux à moder, Deschampsia flexuosa et Luzula albida, et sol colluvial à mull, Mercurialis perennis, Asperula odorata, Impatiens noli-tangere, les paramètres de fertilité des humus sont les suivants : taux d'agrégats, 58 et $85 \mathrm{p} .100$; perméabilité, $3 \mathrm{et} 31 \mathrm{~cm} / \mathrm{h} ; \mathrm{pH}$ eau, 3,5 et 5,4 ; taux de saturation au pH du sol, 10 et 90 p. 100.
\end{abstract}

\section{Introduction}

On sait depuis les travaux de Duvigneaud (1946), puis de Duchaufour (1957, 1960), de Gounot (1959) et d'Emberger et Long (1959) qu'il se crée d'étroites relations entre les associations végétales ou groupes écologiques et les propriétés des sols, plus spécialement celles des humus. La notion de plante indicatrice est donc très utile. On observe ainsi dans des séquences de sols fortement acidifiés, des variations très spectaculaires de la flore herbacée qui paraissent indiquer des divergences stationnelles très fondamentales. Le cas est très fréquent sur les pentes colluvionnées des Vosges cristallines à l'étage montagnard. Les sols bruns colluviaux à flore mésoneutrophile (lamier jaune, mercuriale, parisette, aspérule odorante) sont la plupart du temps associés en chaînes à des sols bruns acides et bruns ocreux qui portent une association végétale toute différente : il s'agit de groupes écologiques dominés par la fétuque des bois ou par l'association canche flexueuse-luzule blanche.

Il existe entre ces sols des différences morphologiques non négligeables, notamment dans l'organisation structurale des humus et dans les modes d'agrégation. Par contre, les caractéristiques du complexe adsorbant et le $\mathrm{pH}$ ne traduisent apparemment que des différences assez faibles.

* Etudiante du DEA de Pédologie, Université de Nancy I. 1976.

Annales des Sciences forestières. - 1978 
Nous avons donc cherché dans ce travail, par une série de déterminations physiques (taux d’agrégats stables, perméabilité) et physico-chimiques (capacité d'échange cationique à plusieurs $\mathrm{pH}$ ), à trouver des relations signifiantes entre les variations de la flore ef les caractéristiques stationnelles.

\section{I. - Description des stations ef analyse des sols}

L'étude que nous présentons a pour cadre quatre stations situées sur un même versant des Hautes Vosges, côté alsacien. Trois des sols retenus sont associés en une chaîne, dite du Robinot, située à $2 \mathrm{~km}$ environ au nord-est du col de Sainte-Marieaux-Mines ; le quatrième sol se trouve à $1 \mathrm{~km}$ à l'est de la chaîne du Robinot, au lieudit Pierre-de-Lusse.

La roche-mère correspond à l'arène du granite des crêtes porphyroïde, mésocrate, à composition de granosyénite (Hameurt, 1967) : quartz (12 à 18 p. 100), feldspath potassique (20 à 55 p. 100), plagioclase à 10 à 20 p. 100 d'anorthite, biotite souvent chloritisée, amphibole et un peu d'apatite. II contient en outre de nombreux filons aplitiques.

Le granite des crêtes a la composition chimique suivante (p. 100) :

$$
\frac{\mathrm{SiO}_{2}}{63,21} \quad \frac{\mathrm{Al}_{2} \mathrm{O}_{3}}{13,63} \quad \frac{\mathrm{Fe}_{2} \mathrm{O}_{3}}{4,88} \quad \frac{\mathrm{MgO}}{4,55} \quad \frac{\mathrm{CaO}}{2,93} \quad \frac{\mathrm{Na}_{2} \mathrm{O}}{2,37} \quad \frac{\mathrm{K}_{2} \mathrm{O}}{6,49} .
$$

La hêtraie-sapinière constitue la formation climacique de l'étage montagnard moyen ; seule la station située au sommet de la toposéquence du Robinot est occupée par une pessière.

Les stations étudiées (tabl. analytique 1) se répartissent le long d'une pente qui s'étend sur environ $100 \mathrm{~m}$ de dénivellation (pente à 30 p. 100).

\section{1. - Station de haut de pente : Pessière à moder et sol brun ocreux (Robinot)}

En haut de pente $(970 \mathrm{~m})$, on observe la canche flexueuse (Deschampsia flexuosa) et la luzule blanche (Luzula albida), espèces acidiphiles, peu exigeantes en bases, pauvres en tous éléments (Duvigneaud et Denayer de Smet, 1970).

Actuellement, la strate herbacée est presque inexistante en raison du peu de lumière que laisse filtrer le peuplement d'épicéas. L'humus est un moder très acide $(\mathrm{pH} 3,5)$, fortement désaturé en bases. Le profil, peu profond, brunifié, à l'horizon (B) de couleur vive, est celui d'un sol brun ocreux.

$A_{00} \quad$ Litière mince d'aiguilles d'Epicéa.

$A_{11} \quad$ Moder brun noir (5 YR 2/2). Aspect compact et mal humifié. Sablo-limoneux. Structure 0-10 cm particulaire massive. Porosité faible. Quelques grosses racines. Peu de cailloux. Grains de quartz blanchis. Limite nette irrégulière.

$A_{12} \quad B$ run rougeâtre (5 YR 3/4). Moins riche en matière organique. Limono-sableux. Structure $10-35 \mathrm{~cm}$ en petits agrégats arrondis friables $(2-3 \mathrm{~mm})$. Porosité moyenne. Présence de cailloux de granite altéré. Quelques infiltrations de matière organique plus sombre (5 YR 3/3) liée aux racines. Transition diffuse et irrégulière avec I'horizon inférieur.

(B) Brun légèrement ocreux (5 YR 4/6). Limono-sableux. Structure en agrégats plus grossiers $35-75 \mathrm{~cm}$ à tendance polyédrique. Texture limono-sableuse. Porosité moyenne. Peu de racines. Peu de cailloux. Quelques infiltrations de matière organique. Transition progressive.

C Arène granitique brun rougeâtre (5 YR 4/4). Sablo-graveleux. Structure faiblement polyédrique. Cailloux abondants de granite altéré. 
2. - Station de mi-pente : Sapinière-hêtraie à mull moder et sol brun acide (Robinot)

A mi-pente, la fétuque (Festuca sylvatica) remplace rapidement la canche ef la luzule : disséminée par touffes en partie haute de la station, elle occupe vers le bas des surfaces de plus en plus grandes; elle se trouve associée, lorsqu'on descend, à des espèces plus exigeantes en bases (Asperula odorata, Lamium galeobdolon, Athyrium filix-femina).

L'humus est à peine moins acide que le précédent $(\mathrm{pH} 3,8)$ mais biologiquement plus actif, de type mull-moder. Par sa position topographique, il reçoit des éléments du sommet de la séquence, mais perd également des matières entraînées plus bas. II demeure encore très désaturé en bases. Le profil, plus profond que le précédent, se range parmi les sols bruns acides:

$A_{00} \quad$ Litière peu épaisse de feuilles de hêtre en surface, puis aiguilles de sapin.

$A_{11} \quad$ Mull à mull-moder brun foncé (5 YR 2/2). Mal structuré : structure en petits grumeaux

0-10 cm friables $(0,5$ à $1 \mathrm{~cm})$. Sablo-limoneux. Bonne porosité. Racines nombreuses fines ou moyennes. Cailloux anguleux peu abondants. Transition progressive ef irrégulière.

$A_{12} \quad$ Moins riche en matière organique. Brun (5 YR 3/3). Sablo-limoneux. Structure en petits

$10-30 \mathrm{~cm}$ agrégats anguleux friables $(1 \mathrm{~cm})$. Porosité moyenne à forte. Quelques cailloux. Présence de quartz blanchis. Racines petites et moyennes. Présence de taches ocres diffuses (2,5 YR 3/3). Transition progressive.

(B) $1 \quad$ Brun jaunâtre (5 YR 4/6) avec passées plus ocres. Sableux. Structure en grumeaux poly$30-80 \mathrm{~cm}$ édriques de $1 \mathrm{~cm}$. Porosité moyenne. Horizon meuble. Quelques cailloux. Racines moyennes. Taches de l'horizon supérieur et inférieur par pédoturbation. Transifion progressive.

(B) $\quad$ Brun rougeâtre plus clair (2,5 YR 4/4). Sablo-graveleux. Structure polyédrique grossière.

$80-100 \mathrm{~cm}$ Plus tassé mais encore poreux. Cailloux assez nombreux. Transition progressive.

(B)/C Brun rougeâtre (2,5 YR 4/4). Sablo-graveleux. Gros polyèdres mal structurés. Nombreux $100-110 \mathrm{~cm}$ cailloux.

C Arène de granite porphyroïde. Nombreux cailloux.

\section{3. - Stations de bas de pente}

a) Sapinière-hêtraie, feuillus divers et sol brun colluvial à mull oligotrophe (Robinot).

En bas de pente $(860 \mathrm{~m})$, près d'un thalweg, la strate herbacée devient très luxuriante et diversifiée.

On retrouve les espèces rencontrées sur le sol brun acide, plus abondantes toutefois, montrant la plus grande activité de l'humus de type mull. On relève également des espèces plus exigeantes en bases et en eau : Paris quadrifolia, Senecio fuchsii, Geranium robertianum, Impatiens noli-tangere.

Par sa situation dans le paysage, le sol de type brun colluvial s'approfondit encore. s'enrichit en bases et en humidité ; le colluvium instable qui caractérise les pentes fortes, entretient une porosite élevée dans les horizons supérieurs du profil.

$A_{00} \quad$ Feuilles de hêtre en décomposition par cellulolyse, puis tapis d'aiguilles de sapin et débris de feuilles de hêtre avec racines très fines.

A 11 Mull brun foncé (5 YR 3/2). Sablo-limoneux. Structure en petits grumeaux arrondis de

$0-10 \mathrm{~cm} \quad 1$ à $2 \mathrm{~mm}$. Très aéré. Porosité forte. Abondant chevelu de petites racines. Quelques cailloux. Transition progressive avec l'horizon inférieur.

$A_{12} \quad$ Moins riche en matière organique (5 YR 3/3). Sablo-limoneux. Structure en grumeaux $10-20 \mathrm{~cm}$ arrondis mais plus grand $(2-4 \mathrm{~mm})$ et plus anguleux. Porosité forte. Racines moins abondantes. Transition progressive.

A/B Même couleur. Sablo-limoneux. Structure en grumeaux polyédriques de plus grande $20-45 \mathrm{~cm}$ taille $(1 \mathrm{~cm})$. Porosité forte. Racines plus grosses. Rares cailloux. Transition progressive. 
(B) $\quad$ Teinte plus claire (5 YR 4/5). Sablo-limoneux. Structure en grumeaux anguleux. Poroperturbé sité bonne mais horizon plus tassé. Taches grisâtres de l'hoziron supérieur par remanie45-80 cm ment. Parallèlement à la pente, elles se présentent sous forme de langues orientées vers l'amont. Racines préférentiellement dans ces zones.

$(B)_{2} \quad$ Brun jaunâtre (5 YR 4/6). Plus sableux. Polyèdres assez émoussés. Plus tassé. Cailloux 80-130 cm fins arrondis. Transition progressive.

C Arène jaunâtre (5 YR 5/4). Graveleux. Nombreux cailloux de granite altéré.

b) Sapinière-hêtraie à mull mésotrophe et sol colluvial (Pierre-de-Lusse).

La station se caractérise par l'abondance de la mercuriale (Mercurialis perennis) qui devient envahissante, associée à l'aspérule (Asperula odorata) et la balsamine (Impatiens noli-tangere).

L'humus structuré en grumeaux bien développés et solides contient suffisamment de bases pour être classé parmi les mull mésotrophes. Le profil de type AC est celui d'un sol colluvial.

$A_{00}$ Litière pratiquement inexistante.

$A_{1}$ Mull de couleur brun foncé (5 YR 2/2). Structure en petits grumeaux arrondis, très mobiles, solides, infiltrés entre le feutrage de racines et les nombreux cailloux et blocs de granite de toutes tailles.

Tous les sols étudiés ont une texture sableuse (tabl. 1) ; les teneurs en argile et en produits amorphes augmentent du bas vers le haut des profils, sans redistribution

\section{TABLEAU 1}

Caractéristiques chimiques et physiques des horizons secs à l'air, tamisés à $2 \mathrm{~mm}$, de quatre sols associés en chaine

Chemical and physical characteristics of horizons samples

(0-2 $\mathrm{mm}$ air dried fraction) from four soils forming a catena

\begin{tabular}{|c|c|c|c|c|c|c|c|c|c|c|c|c|c|c|c|c|}
\hline & \multirow{2}{*}{$\begin{array}{l}\text { Prof. } \\
\mathrm{cm}\end{array}$} & \multirow{2}{*}{ Hor. } & \multicolumn{5}{|c|}{$\begin{array}{c}\text { Granulométrie } \\
\text { p. } 100\end{array}$} & \multirow{2}{*}{$\begin{array}{l}\text { M. O. } \\
\text { p. } 100\end{array}$} & \multirow{2}{*}{$\mathrm{C} / \mathrm{N}$} & \multirow{2}{*}{$\begin{array}{l}\mathrm{pH} \\
\text { eau }\end{array}$} & \multirow{2}{*}{\multicolumn{2}{|c|}{$\frac{\mathrm{S} C \mathrm{~T}}{\mathrm{me} / 100 \mathrm{~g}}$}} & \multirow{2}{*}{$\begin{array}{l}100 \\
\mathrm{~S} / \mathrm{T}\end{array}$} & \multicolumn{3}{|c|}{$\begin{array}{l}\text { Eléments } \\
\text { amorphes p. } 1000\end{array}$} \\
\hline & & & S. G. & S. F. & L. G. & L. F. & A. & & & & & & & $\mathrm{Fe}$ & $\mathrm{Al}$ & Si \\
\hline $\begin{array}{l}\text { Sol brun } \\
\text { ocreux }\end{array}$ & $\begin{array}{r}0-10 \\
10-35 \\
35-75 \\
>75\end{array}$ & $\begin{array}{l}A_{0} A_{11} \\
A_{12} \\
(B) \\
C\end{array}$ & $\begin{array}{l}46,1 \\
55,1 \\
56,2 \\
58,1\end{array}$ & $\begin{array}{r}6,5 \\
10,6 \\
10,7 \\
12,7\end{array}$ & $\begin{array}{l}3,9 \\
4,4 \\
5,9 \\
6,1\end{array}$ & $\begin{array}{l}19,7 \\
15,5 \\
14,7 \\
14,6\end{array}$ & $\begin{array}{r}23,8 \\
14,4 \\
12,5 \\
8,5\end{array}$ & $\begin{array}{r}32,2 \\
11,7 \\
4,2 \\
1,6\end{array}$ & $\begin{array}{l}18,9 \\
17,8 \\
18,9 \\
23,2\end{array}$ & $\begin{array}{l}3,5 \\
4,0 \\
4,3 \\
4,5\end{array}$ & $\begin{array}{l}1,27 \\
0,50 \\
0,30 \\
0,31\end{array}$ & $\begin{array}{l}35,6 \\
20,7 \\
15,3 \\
11,3\end{array}$ & $\begin{array}{l}3,6 \\
2,4 \\
2,0 \\
2,7\end{array}$ & $\begin{array}{r}11,0 \\
11,9 \\
9,3 \\
5,7\end{array}$ & $\begin{array}{l}4,1 \\
6,0 \\
7,1 \\
6,5\end{array}$ & $\begin{array}{l}0,5 \\
0,5 \\
0,5 \\
0,9\end{array}$ \\
\hline $\begin{array}{l}\text { Sol brun } \\
\text { acide }\end{array}$ & $\begin{array}{c}0-10 \\
10-30 \\
30-80 \\
80-100 \\
100-115 \\
>115\end{array}$ & $\begin{array}{l}A_{11} \\
A_{12} \\
(B)_{1} \\
(B)_{2} \\
(B) / C \\
C\end{array}$ & $\begin{array}{l}57,0 \\
56,6 \\
59,5 \\
53,0 \\
57,7 \\
63,7\end{array}$ & \begin{tabular}{|l|}
10,6 \\
12,7 \\
11,4 \\
13,9 \\
12,7 \\
11,3
\end{tabular} & $\begin{array}{l}5,7 \\
5,0 \\
4,8 \\
6,0 \\
5,4 \\
4,1\end{array}$ & $\begin{array}{l}11,0 \\
11,5 \\
11,4 \\
13,6 \\
12,9 \\
10,3\end{array}$ & $\begin{array}{l}15,7 \\
14,2 \\
12,9 \\
13,5 \\
11,3 \\
10,6\end{array}$ & $\begin{array}{r}12,3 \\
6,2 \\
2,4 \\
1,6 \\
1,5 \\
0,9\end{array}$ & $\begin{array}{l}15,8 \\
17,1 \\
20,0 \\
16,4 \\
21,1 \\
21,3\end{array}$ & $\begin{array}{l}3,8 \\
4,1 \\
4,3 \\
4,7 \\
4,6 \\
4,9\end{array}$ & $\begin{array}{l}1,37 \\
0,72 \\
0,60 \\
0,43 \\
0,37 \\
0,47\end{array}$ & $\begin{array}{l}19,3 \\
17,4 \\
17,1 \\
13,2 \\
13,3 \\
10,6\end{array}$ & $\begin{array}{l}7,1 \\
4,1 \\
3,5 \\
3,4 \\
2,8 \\
4,4\end{array}$ & $\begin{array}{l}7,6 \\
9,2 \\
9,5 \\
8,1\end{array}$ & $\begin{array}{l}4,0 \\
5,2 \\
5,1 \\
5,4 \\
\\
5,2\end{array}$ & $\begin{array}{l}0,8 \\
0,9 \\
0,5 \\
0,5 \\
0,7\end{array}$ \\
\hline $\begin{array}{l}\text { Sol brun } \\
\text { colluvial }\end{array}$ & $\begin{array}{c}0-10 \\
10-20 \\
20-45 \\
45-80 \\
80-130 \\
>130\end{array}$ & $\begin{array}{l}A_{11} \\
A_{19} \\
A / B \\
(B){ }_{1} \\
(B)_{2} \\
C\end{array}$ & $\begin{array}{l}60,2 \\
59,0 \\
53,5 \\
54,5 \\
64,8 \\
64,9\end{array}$ & \begin{tabular}{r|}
8,4 \\
8,5 \\
9,5 \\
10,2 \\
6,9 \\
8,3
\end{tabular} & $\begin{array}{l}5,3 \\
5,5 \\
5,3 \\
5,8 \\
5,8 \\
5,5\end{array}$ & $\begin{array}{l}11,1 \\
12,4 \\
12,4 \\
16,0 \\
13,4 \\
14,8\end{array}$ & $\begin{array}{r}15,0 \\
14,6 \\
13,3 \\
13,5 \\
9,9 \\
6,5\end{array}$ & $\begin{array}{r}12,0 \\
7,2 \\
3,6 \\
1,6 \\
1,4 \\
0,9\end{array}$ & $\begin{array}{l}15,8 \\
14,4 \\
15,1 \\
16,4 \\
17,7 \\
17,9\end{array}$ & $\begin{array}{l}4,5 \\
4,6 \\
4,1 \\
4,7 \\
4,8 \\
4,9\end{array}$ & $\begin{array}{l}5,00 \\
4,54 \\
1,10 \\
0,65 \\
0,54 \\
0,46\end{array}$ & \begin{tabular}{|r|}
21,9 \\
21,5 \\
15,0 \\
13,8 \\
13,4 \\
8,6
\end{tabular} & $\begin{array}{r}22,8 \\
21,1 \\
7,3 \\
4,6 \\
3,9 \\
5,3\end{array}$ & $\begin{array}{l}8,5 \\
9,0 \\
9,3 \\
8,9 \\
8,8 \\
5,9\end{array}$ & $\begin{array}{l}5,0 \\
5,1 \\
5,5 \\
6,6 \\
6,7 \\
5,2\end{array}$ & $\begin{array}{l}0,7 \\
0,7 \\
0,5 \\
0,6 \\
0,7 \\
0,7\end{array}$ \\
\hline $\begin{array}{c}\text { Sol } \\
\text { colluvial }\end{array}$ & $0-10$ & $A_{1}$ & 65,8 & 7,4 & 4,9 & 9,7 & 12,2 & 6,0 & 15,0 & 5,3 & 8,12 & 14,3 & 57,0 & 4,5 & 2,3 & 0,3 \\
\hline
\end{tabular}


apparente d'argile, de fer, d'aluminium. Les descriptions faites ci-dessus ef l'analyse plus approfondie des structures et microstructures réalisée par Selino (1976) rendent compte de l'accroissement progressif de l'agrégation : 1) verticalement au sein d'un même profil, 2) latéralement depuis le sol brun ocreux jusqu'au sol colluvial.

\section{II. - Résultats}

A. - Caractéristiques structurales : taux d'agrégats et perméabilité (†abl. 2)

Les tests de mesure d'agrégats stables et de perméabilité (Henin et al., 1960) indiquent que la stabilité structurale augmente régulièrement du sol brun ocreux au sol

\section{TABLEAU 2}

Stabilité structurale et perméabilité des horizons de quatre sols associés en chaîne

Structural stability and permeability of horizons samples from four soils forming a catena

\begin{tabular}{|c|c|c|c|c|c|c|}
\hline & \multirow{2}{*}{ Horizon } & \multirow{2}{*}{$\begin{array}{c}\text { Prof. } \\
\mathrm{cm}\end{array}$} & \multicolumn{3}{|c|}{ Taux d'agrégats stables p. 100 du sol } & \multirow{2}{*}{$\underset{\mathrm{cm} / \mathrm{h}}{\mathrm{K}}$} \\
\hline & & & Alcool p. 100 & Benzène p. 100 & Air p. 100 & \\
\hline Sol brun ocreux & $\begin{array}{l}A_{11} \\
A_{12} \\
(B) \\
C\end{array}$ & $\begin{array}{r}0-10 \\
10-35 \\
35-75 \\
>75\end{array}$ & $\begin{array}{l}69,1 \\
72,3 \\
68,0 \\
65,8\end{array}$ & $\begin{array}{l}63,3 \\
72,1 \\
66,4 \\
57,1\end{array}$ & $\begin{array}{l}58,0 \\
70,4 \\
67,2 \\
69,0\end{array}$ & $\begin{array}{l}2,6 \\
4,0 \\
4,5 \\
0,2\end{array}$ \\
\hline Sol brun acide & $\begin{array}{l}A_{11} \\
A_{12} \\
(B)_{1} \\
(B)_{2} \\
(B) / C \\
C\end{array}$ & $\begin{array}{c}0-10 \\
10-30 \\
30-80 \\
80-100 \\
100-115 \\
>115\end{array}$ & $\begin{array}{l}63,2 \\
71,3 \\
67,8 \\
69,8 \\
65,7 \\
71,4\end{array}$ & $\begin{array}{l}65,8 \\
74,6 \\
69,3 \\
64,2 \\
61,8 \\
57,8\end{array}$ & $\begin{array}{l}65,0 \\
71,8 \\
76,0 \\
72,1 \\
72,9 \\
69,8\end{array}$ & $\begin{array}{r}7,5 \\
4,5 \\
12,3 \\
3,2 \\
0,2 \\
1,4\end{array}$ \\
\hline Sol brun colluvial & $\begin{array}{l}A_{11} \\
A_{12} \\
A / B \\
(B)_{1} \\
(B)_{2} \\
C\end{array}$ & $\begin{array}{c}0-10 \\
10-20 \\
20-45 \\
45-85 \\
85-130 \\
>130\end{array}$ & $\begin{array}{l}76,4 \\
77,4 \\
69,4 \\
70,3 \\
76,4 \\
69,6\end{array}$ & $\begin{array}{l}77,4 \\
70,3 \\
70,8 \\
63,0 \\
62,4 \\
58,0\end{array}$ & $\begin{array}{l}80,0 \\
77,4 \\
74,6 \\
69,4 \\
78,5 \\
68,0\end{array}$ & $\begin{array}{r}18,7 \\
13,4 \\
2,0 \\
0,5 \\
3,4 \\
1,5\end{array}$ \\
\hline Sol colluvial & $A_{1}$ & $0-10$ & 82,9 & 86,3 & 85,0 & 31,0 \\
\hline
\end{tabular}

colluvial. Cette variation latérale dans la toposéquence est observée pour tous les horizons et notamment pour les humus. Le minimum et le maximum de taux d'agrégats stables (prétraitement air) correspondent en effet aux deux types d'humus rencontrés aux deux extrémités de la chaîne : le moder très acide du sommet éluvié avec 58 p. 100 et le mull mésotrophe du bas illuvié avec 85 p. 100. De même, les valeurs extrêmes des perméabilités reflètent le mode d'arrangement des constituants organiques et minéraux : la structure est soit prise en masse dans le moder du sol brun ocreux (faible perméabilité), soit agrégée et poreuse dans le mull du sol colluvial (forte perméabilité). 


\section{B. - Capacité d'échange cationique et taux de saturation à $\mathrm{pH} 7$ et au $\mathrm{pH}$ du sol}

Il est très important de noter tout d'abord que le sol se comporte comme un échangeur de cations dont la capacité totale d'échange (CEC) est variable, en particulier avec le $\mathrm{pH}$ du sol, et, au laboratoire, avec le $\mathrm{pH}$ de la solution d'échange qui permet de mesurer la CEC.

Rappelons brièvement l'origine de la capacité d'échange du complexe argilohumique du sol. Les colloïdes du sol présentent une charge de surface compensée par une charge équivalente et de signe opposé, accumulée au contact de la surface des particules colloïdales. Ainsi, la charge électronégative des minéraux argileux et de la matière organique est équilibrée par des cations compensateurs, dits échangeables, parce qu'accessibles aux échanges réversibles, et dont l'ensemble constitue la CEC.

L'origine de la charge de surface peut être structurale : ce sont les substitutions isomorphiques qui créent dans les réseaux cristallins des phyllites (montmorillonites) des charges négatives, dites charges permanentes.

D'autres mécanismes plus complexes font apparaître des charges dites variables, dépendant notamment des ions $\mathrm{H}^{+}$et $\mathrm{OH}^{-}$présents dans la solution du sol, c'est-à-dire du $\mathrm{pH}$. Il s'agit de la dissociation protonique des colloïdes organiques et de la dissociation amphotérique des sites superficiels des silicates qui induisent des charges négatives, fonction $\mathrm{du} \mathrm{pH}$.

\section{Colloïdes organiques : $\mathrm{RCOOH}+\mathrm{OH}^{-} \rightleftarrows \mathrm{RCOO}^{-}+\mathrm{HOH}$.}

Silicates

$$
\begin{aligned}
& : \mathrm{SiOH}+\mathrm{OH}^{-} \rightleftarrows \mathrm{SiO}^{-}+\mathrm{HOH} \\
& : \mathrm{AlOH}+\mathrm{OH}^{-} \rightleftarrows \mathrm{AlO}^{-}+\mathrm{HOH} .
\end{aligned}
$$

De même, la surface des oxydes hydratés, par adsorption et désorption d'ions $\mathrm{H}^{+}$et $\mathrm{OH}^{-}$, acquiert des charges variables positives ou négatives, fonctions du $\mathrm{pH}$.

Ainsi, pour l'ensemble du complexe d'échange d'un sol, la charge totale $\left(\sigma_{t}\right)$ est la somme des charges permanentes $\left(\sigma_{\mathrm{p}}\right)$ et des charges variables $\left(\sigma_{\mathrm{v}}\right)$. Cette charge totale $\left(\sigma_{t}\right)$ correspond à la capacité d'échange et peut s'annuler pour une certaine valeur du $\mathrm{pH}$. Cette valeur du $\mathrm{pH}$ est appelée ZPC (point de charge nulle).

On démontre qu'il existe une relation de la forme

$$
\sigma_{\mathrm{t}}=\mathrm{a}+\mathrm{k}(\mathrm{ZPC}-\mathrm{pH}) \text {. }
$$

Le ZPC est généralement situé vers des valeurs de $\mathrm{pH}$ voisines de 3 et ainsi, quand on s'éloigne de ce point de charge nulle, la charge, et donc la capacité d'échange du sol, augmentent avec le $\mathrm{pH}$. Ce phénomène de variation de la charge avec le $\mathrm{pH}$ est tout particulièrement marquée dans les sols humifères et dans les sols riches en hydroxydes.

La somme $\mathrm{S}$ des cations échangeables alcalins et alcalino-terreux fixés sur le complexe adsorbant est une donnée importante qui dépend de l'équilibre entre sol et solutions et, bien entendu, de la CEC. L'écart entre la CEC et $S$ est en fait représenté par les ions $\mathrm{Al}^{+++}$et $\mathrm{H}^{+}$qui sont d'autant plus abondants que le sol est désaturé.

La nutrition minérale des plantes est fortement influencée par la proportion réelle existant sur le complexe d'échange entre cations échangeable $\mathrm{S}$ et les ions $\mathrm{Al}++++\mathrm{H}^{+}$. Il est bien clair que la détermination analytique du taux de saturation S/CEC n'aura 
TABLEAU 3

Cations échangeables, somme des bases échangeable (S), capacité d'échange cationique et taux de saturation à $\mathrm{pH} 7$ ef au $\mathrm{pH}$ du sol (chaîne du Robinot) Exchangeable cations, total exchangeable bases (S), cations exchange capacity and degree of saturation at 7 and soil pH (soils catena)

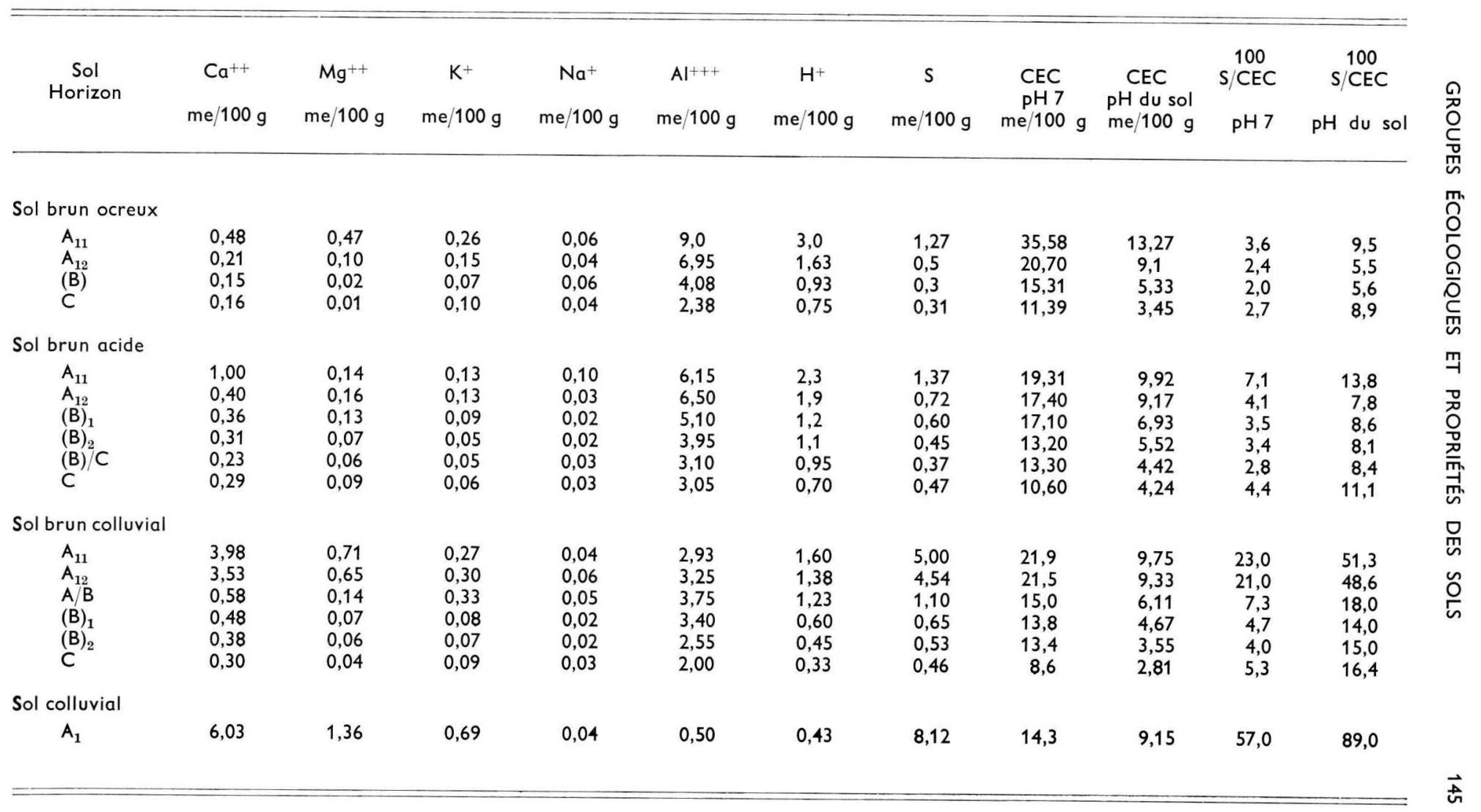


de signification écologique précise que si la capacité totale d'échange est mesurée au pH du sol (Chirita, 1971 ; Chirita et lonescu, 1973 ; Chirita, 1974). On détermine ainsi les caractéristiques ioniques dans les conditions de $\mathrm{pH}$ qui règlent les échanges entre le complexe adsorbant, les solutions du sol et la rhizosphère des végétaux.

Avant de commenter les résultats, précisons les modalités de détermination de la capacité d'échange cationique au $\mathrm{pH}$ du sol. On procède à la saturation des charges électronégatives actuelles du sol par l'ion $\mathrm{K}^{+}$d'une solution $\mathrm{N}$ de chlorure de potassium non tamponnée, qui s'ajuste à un $\mathrm{pH}$ très proche de celui du sol. On opère par percolation de $4 \mathrm{~g}$ d'échantillon par $100 \mathrm{ml}$ de solution. On conserve ce premier percolat (Coleman et al., 1959 ; Espiau et Peyronel, 1976).

Dans le premier percolat, on détermine les cations échangeables $\mathrm{Ca}^{++}, \mathrm{Mg}^{++}, \mathrm{Na}^{+}, \mathrm{Al}^{+++}, \mathrm{H}^{+}$, soit par absorption atomique, soit par titrimétrie. $\mathrm{K}^{+}$échangeable est obtenu par percolation d'un deuxième échantillon de sol par l'acétate d'ammonium qui sert à la mesure de la CEC à pH 7 .

La somme $\left(\mathrm{CA}^{++}, \mathrm{Mg}^{++}, \mathrm{K}^{+}, \mathrm{Na}^{+}\right)+\left(\mathrm{Al}^{+++}, \mathrm{H}^{+}\right)$détermine la $\mathrm{CEC}$ au $\mathrm{pH}$ du sol.

Les résultats présentés dans le tableau 3 rendent compte de l'état du complexe adsorbant. La CEC varie le long de la toposéquence essentiellement en fonction de la teneur en matière organique, comme le démontre la forte augmentation de la CEC à $\mathrm{pH} 7$ par rapport à la $\mathrm{CEC}$ au $\mathrm{pH}$ du sol des horizons $\mathrm{A}_{11}$ les plus humifères. La figure 1 représente sous forme de cercles, la $\mathrm{CEC} \mathrm{pH} 7$ et $\mathrm{pH}$ du sol (proportionnelle au rayon) et la teneur relative de chaque cation d'échange (proportionnelle au secteur circulaire).

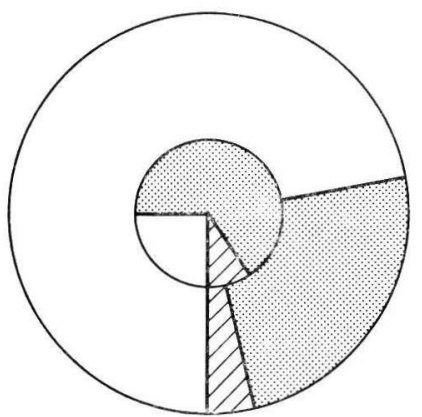

Sol brun ocreux $A_{11}$

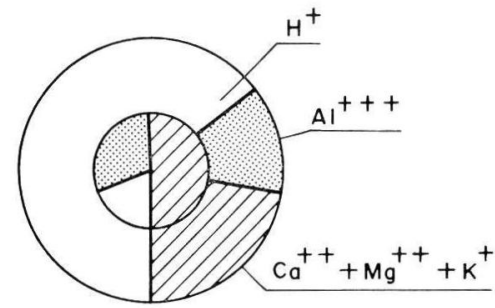

Sol brun colluvial $A_{11}$

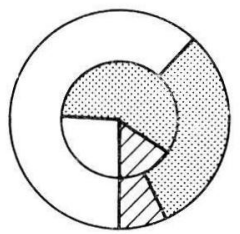

Sol brun acide $A_{11}$



Sol colluvial $A_{1}$ 10 me de CEC pour le rayon.

FIG. 1. - Etat du complexe adsorbant à pH 7 (cercle extérieur) et au pH du sol (cercle intérieur) : rayon proportionnel à la CEC.

Base saturation at $7 \mathrm{pH}$ (outer circle) and soil $\mathrm{pH}$ (inner circle) of $A_{1}$ horizons of four soils forming a catena (radius represents CEC). 
Les cations qui équilibrent la CEC au $\mathrm{pH}$ du sol sont fournis en majeure partie : 1) par $\mathrm{Al}^{+++}$et $\mathrm{H}^{+}$pour les sols bruns ocreux et bruns acides, situés en haut de la toposéquence, 2) par les ions basiques alcalino-terreux et alcalins, surtout $\mathrm{Ca}^{++}$pour les sols bruns colluviaux analysés en bas de cette même pente. La démonstration est particulièrement claire dans les horizons $A_{1}$ (ou $A_{11}$ ). Dans les sols à humus de type moder et mull-moder, le complexe adsorbant reste fortement désaturé en bases quel que soit le $\mathrm{pH}$ de référence : l'ion responsable au premier chef de la désaturation étant le métal $\mathrm{Al}^{+++}$au $\mathrm{pH}$ du sol et le proton $\mathrm{H}^{+}$à $\mathrm{pH}$ 7. Par contre, les sols à humus de type mull des stations situées en bas de pente, encore fortement désaturés à $\mathrm{pH} 7$, se comportent comme des sols mésotrophes (sol brun colluvial) et eutrophes (sol colluvial) au $\mathrm{pH}$ du sol.

\section{III. - Discussion et conclusion}

L'analyse des propriétés physiques - taux d'agrégats stables, perméabilité ef l'estimation du taux de saturation calculé à partir de la capacité d'échange cationique au pH du sol, différencient de façon hautement significative les sols brunifiés de type brun ocreux, brun acide, brun colluvial et colluvial rencontrés sur roche cristalline à l'étage montagnard. Le climat froid qui règne en montagne augmente le caractère humifère des sols. Des composés humiques pseudosolubles imprègnent les profils, attestant d'une podzolisation climatique potentielle (Bruckert et al., 1975) ; ce caractère atténue les différences morphologiques et accroît anormalement la capacité d'échange cationique mesurée à $\mathrm{pH} 7$ : dans ces conditions, les sols brunifiés ont tous des taux de saturation faibles, alors que leur fertilité, indiquée par le niveau de production sylvicole et par la composition des groupes écologiques, est manifestement très varicble.

Comme on vient de le voir, les caractéristiques électro-ioniques réelles du sol apparaissent bien plus nettement quand on les mesure au $\mathrm{pH}$ du sol. Un meilleur choix des paramètres édaphiques de fertilité permet de justifier la répartition des groupes écologiques et notamment la présence de plantes exigeantes en calcium, comme la mercuriale, sur des sols et des humus acides ; les correspondances établies sont rassemblées dans le tableau 4. Ces premiers résultats encourageants constituent l'amorce d'une série d'investigations que nous comptons poursuivre sur un plus grand nombre de stations, en vue de préciser, en fonction de chaque groupe écologique rencontré dans les Vosges, les intervalles de variation des paramètres édaphiques retenus.

L'interprétation physiologique est fondamentale au niveau de la végétation (Wacquant, 1969) : les plantes neutrophiles (et hygrophiles) se révèlent peu tolérantes à $\mathrm{Al}^{+++}$et exigent une forte saturation en ions alcalino-terreux. Au contraire, les acidiphiles strictes sont fortement tolérantes à la saturation aluminique. Les plantes des mull acides, et la fétuque en particulier, ont une très réelle plasticité et tolérance à l'ion aluminium.

Les exigences d'ordre chimique ne sont pas les seules à intervenir dans la différenciation des groupes écologiques. La répartition des espèces végétales dépend également des propriétés physiques qui découlent de l'organisation des constituants du sol en unités structurales plus ou moins favorables à la circulation de l'eau et de l'air, c'est-à-dire plus ou moins « fertiles physiquement » (Rotini et Séqui, 1974).

L'activité biologique du milieu est sans doute étroitement dépendante de ces caractéristiques, et comme l'ont montré les travaux de Duchaufour et al. (1971), 
Correspondance entre groupes écologiques et paramètres de fertilité physique et chimique des humus de quatre sols associés en chaine Relationships between ecological groups (plants associations) and humus physico-chemical fertility parameters of four soils forming a catena

\begin{tabular}{|c|c|c|c|c|c|c|}
\hline \multicolumn{3}{|c|}{ Caractères des stations } & \multicolumn{4}{|c|}{ Paramètres de fertilité des humus (horizon $A_{1}$ ) } \\
\hline Végétation & Localisation & Type de sol & $\begin{array}{c}\text { Taux d'agrégats } \\
\text { air p. } 100\end{array}$ & $\begin{array}{l}\text { Perméabilité } \\
\mathrm{K} \mathrm{cm} / \mathrm{h}\end{array}$ & $\begin{array}{l}\mathrm{pH} \\
\text { eau }\end{array}$ & $\begin{array}{c}\text { Taux de saturation } \\
\text { au } \mathrm{pH} \text { du sol }\end{array}$ \\
\hline $\begin{array}{l}\text { Pessière } \\
\text { Deschampsia flexuosa } \\
\text { Luzula albida }\end{array}$ & $\begin{array}{l}\text { Haut de pente } \\
\text { Altitude : } 970 \mathrm{~m} \\
\text { Exposition SSE }\end{array}$ & $\begin{array}{l}\text { Sol brun ocreux } \\
\text { peu profond à moder } \\
\text { très désaturé }\end{array}$ & 58 & 2,6 & 3,5 & 9,5 \\
\hline $\begin{array}{l}\text { Sapinière-Hêtraie } \\
\text { Festuca sylvatica } \\
\text { Luzula albida } \\
\text { Asperula odorata } \\
\text { Lamium galeobdolon } \\
\text { Athyrium filix femina } \\
\text { Geranium robertianum }\end{array}$ & $\begin{array}{l}\text { Mi-pente } 30 \text { p. } 100 \\
\text { Altitude : } 930 \mathrm{~m} \\
\text { Exposition SSE }\end{array}$ & $\begin{array}{l}\text { Sol brun acide } \\
\text { moyennement profond, à } \\
\text { mull-moder désaturé }\end{array}$ & 65 & 7,5 & 3,85 & 13,8 \\
\hline $\begin{array}{l}\text { Sapinière-Hêtraie } \\
\text { Festuca sylvatica } \\
\text { Asperula odorata } \\
\text { Impatiens noli-tangere } \\
\text { Lamium galeobdolon } \\
\text { Geranium robertianum } \\
\text { Oxalis acetosella } \\
\text { Paris quadrifolia } \\
\text { Senecio fuchsii } \\
\text { Mercurialis perennis }\end{array}$ & $\begin{array}{l}\text { Bas de pente } \\
\text { Thalweg } \\
\text { Altitude : } 860 \mathrm{~m} \\
\text { Exposition SSE }\end{array}$ & $\begin{array}{l}\text { Sol brun colluvial } \\
\text { très profond à mull mé- } \\
\text { sotrophe au pH du sol } \\
\text { bien agrégé, } \\
\text { frais }\end{array}$ & 80 & 18,7 & 4,5 & 51,3 \\
\hline $\begin{array}{l}\text { Sapinière-Hêtraie } \\
\text { Mercurialis perennis } \\
\text { Asperula odorata } \\
\text { Impatiens noli-tangere } \\
\text { Lalium galeobdolon } \\
\text { Festuca sylvatica }\end{array}$ & $\begin{array}{l}\text { Pente } 40 \text { p. } 100 \\
\text { Altitude : } 650 \mathrm{~m} \\
\text { Exposition S }\end{array}$ & $\begin{array}{l}\text { Sol colluvial } \\
\text { Mull eutrophe bien agré- } \\
\text { gé, frais, profond entre } \\
\text { les cailloux }\end{array}$ & 85 & 31,1 & 5,35 & 89,0 \\
\hline
\end{tabular}
Altitude : $650 \mathrm{~m}$ Exposition $\mathrm{S}$
Mull eutrophe bien agréé, frais, profond entr es cailloux 
l'alimentation azotée est beaucoup mieux assurée dans les milieux colluviaux qui se révèlent à comportement eutrophe.

En conclusion, les propriétés physico-chimiques qui naissent de l'interaction entre les constituants organiques et minéraux des sols, expliquent quelques divergences fondamentales de fonctionnement des écosystèmes étagés le long des pentes des Hautes Vosges cristallines et notamment, les divergences fondamentales dans le pédoclimat, l'activité biologique générale et les cycles biogéochimiques.

Reçu pour publication en décembre 1977.

\section{Summary}

\section{Relationships between plants associations and physico-chemical characteristics of soils in a catena of crystalline mountains Vosges (France)}

Physical (structure stability, permeability) and chemical characteristics (cation exchange capacity at different $\mathrm{pH}$ ) of four soils forming a catena along a mountain crystalline slope (mountain floor of Vosges France) were considered to determine relationships between plant association (ecological group) and such forest soils (brown ochreous soil : typic dystrochrept ; brown acid soil : typic dystrochrept ; brown colluvial soil : typic haplumbrept; colluvial soil : typic haplumbrept).

Significant relationship appeared so that humus characteristics such as structural stability (degree of aggregates), permeability, $\mathrm{pH}$, and degree of saturation were the lower (respectively $58 \mathrm{p} .100$; $2,6 \mathrm{~cm} / \mathrm{h} ; 3,5 ; 9,5)$ in the less mature soil (brown ochreous soil with a moder humus and Deschampsia flexuosa, Luzula albida) and the higher (respectively 85 p. $100 ; 31,1 \mathrm{~cm} / \mathrm{h} ; 5,3 ; 89,0$ ) in the younger soil (colluvial soil with Mercurialis perennis, Asperula odorata, Lamium galeobdolon, Fesfuca sylvatica).

Characteristics of acid brown soil in such catena were just between these values.

\section{Zusammenfassung}

Beziehungen zwischen okologischen Standortseinheiten und den physikalischen und chemischen Eigenschaften der Braunerdeboden im kristallinem Gebiet der Vogensen

Die Autoren weisen eine Reihe von physischen (prozentsatz stabiler Agregate Durchdringlichkeit und chemisch physischen (KUK zu verschiedenen $\mathrm{pH}$ ) Bestimmungen hervor, die äusserst bedeutungsvolle Verbindungen zwischen Vegetation (ökologische Gruppen) und « braune saure Colluvial oder ockerartige Böden " feststellen, die man auf einen selben Haug auf Kristallinsteine der Vogesenbergstufe findet.

Für die zwei Extremen, undswar der braun ockerartige moder Boden mit Deschampsia flexuosa und Luzula albida, und auf den colluvial mull Boden mit Mercurialis perennis, Asperula odorata und Impatiens noli-tangere, sind die Fruchtbarkeitsparameter des Humus die folgenden :

- prozent Agregate 58 und 85 p. 100 ; Durchdringlichkeit 3 und $31 \mathrm{~cm} / \mathrm{st}$;

- Wasser pH 3,5 und 5,4; Sättigungsprozent zum Boden pH 10 und 90 p. 100.

\section{Références bibliographiques}

BRUCKERT S., BRETHES A., SOUCHIER B., 1975. Humification et distribution des complexes organo-métalliques des sols brunifiés et podzolisés. C. R. Acad. Sc., Paris, 280 D, 1237-1240.

CHIRITA C. D., 1971. Cercetari asupra starii de aciditate si saturatie in baze a solurilor acide. Anal. Inst. si Cercetari Pedol., 38.

CHIRITA C. D., IONESCU M., 1973. Conditiile actuale si potentiale de aciditate si saturatie in baze a solurilor acide, din punct de vedere fizico-chimic si ecologic. Stiinte solului, 11 (2), 19-31.

CHIRITA C. D., 1974. Ecopedologie cu baze de pedologie generale. Ceres Ed., Bucarest, 296-330.

COLEMAN N. T., WEED S. B., MCCRACKEN R. J., 1959. Cation-exchange capacity and exchangeable cations in Piemont soils of North Carolina. Soil Sci. Soc. Amer. Proc., 23, 146-149. 
DUCHAUFOUR Ph., 1957. Tableaux descriptifs et analytiques des sols. Editions de l'Ecole Nationale des Eaux et des Forêts, Nancy, 87 p.

DUCHAUFOUR Ph., 1960. Stations, types d'humus et groupements écologiques. Rev. Forest fr., 7, 484-494.

DUChaufour Ph., BALANDREAU J., QUELEN Denise, 1971. Minéralisation de l'azote dans deux types de sols bruns acides vosgiens. Bull. E.N.S.A. Nancy, XIII (1), 3-6.

DUVIGNEAUD P., 1946. La variabilité des associations végétales. Bull. Sté Royale de Botan. de Belgique, LXXVIII, p. 107.

DUVIGNEAUD P., DENAYER DE SMET S., 1970. Phyłogéochimie des groupes écosociologiques de Haute Belgique. I. Essai de classification phytogéochimique des espèces herbacées. Oecol. Plant., 5 (1), 1-31.

EMBERGER L., LONG G., 1959. Orientation actuelle au service de la C.G.V. de la cartographie phytosociologique appliquée. Bull. Serv. Carte phytogéographique, IV (2), 119-146.

ESPIAU P., PEYRONEL A., 1976. L'acidité d'échange dans les sols. Méthode de détermination de l'aluminium échangeable et des protons échangeables. Sc. du sol, 3, 161-175.

GOUNOT M., 1959. L'exploitation mécanographique des relevés pour la recherche des groupes écologiques. Bull. Serv. Carte phytogéographique, IV, 2, 147-177.

HAMEURT J., 1967. Les terrains cristallins et cristallophylliens du versant occidental des Vosges moyennes. Thèse Fac. Sc. Nancy. Mém. Serv. Carte Géol. Als. Lorr., 26, 402 p.

HENIN S., GRAS R., MONNIER G., 1960. Le profil cultural. Masson et Cie ed., Paris, 332 p.

ROTINI O. T., SEQUI P., 1974. Progrès réalisés dans la fertilité du sol et nutrition des plantes. Geoderma, 12, 331-346.

SELINO D., 1976. Genèse et évolution de la structure dans la chaîne de sols du Robinot (Haut-Rhin). D.E.A. Université Nancy I.

WACQUANT J. P., 1969. Adsorption, absorption cationique préférentielle et écologie végétale. Bull. Soc. Franç. Physiol. Vég., 15, 237-251. 